\title{
A Neural Network Model for the Self-Organization of Cortical Grating Cells
}

\author{
Christoph Bauer ${ }^{\mathrm{a}}$, Thomas Burger ${ }^{\mathrm{a}}$, Martin Stetter ${ }^{\mathrm{b}}$, Elmar W. Langa,* \\ a Institut für Biophysik, Universität Regensburg, D-93040 Regensburg, Germany. \\ Fax: +49941943 2479. E-mail: elmar.lang@biologie.uni-regensburg.de \\ b FR2-1, Informatik, TU Berlin, D-10587 Berlin, Germany \\ * Author for correspondence and reprint requests
}

Z. Naturforsch. 55c, 282-291 (2000); received July 5/December 10, 1999

Self-Organization, Nonlinearities, Visual Cortex, (Anti-)Hebbian Learning, Lateral Plasticity

A neural network model with incremental Hebbian learning of afferent and lateral synaptic couplings is proposed,which simulates the activity-dependent self-organization of grating cells in upper layers of striate cortex. These cells, found in areas V1 and V2 of the visual cortex of monkeys, respond vigorously and exclusively to bar gratings of a preferred orientation and periodicity. Response behavior to varying contrast and to an increasing number of bars in the grating show threshold and saturation effects. Their location with respect to the underlying orientation map and their nonlinear response behavior are investigated. The number of emerging grating cells is controlled in the model by the range and strength of the lateral coupling structure.

\section{Introduction}

The primary visual cortex is an intensely studied information processing module of the brain. Extensive neurophysiological investigations have accumulated a considerable body of knowledge about the visual system of vertebrates (Kandel et al., 1996). Early stages of information processing extract simple features mainly from the visual input patterns. Recently Von der Heydt et al. (1992), however, discovered a new type of cortical cells extracting complex features like oriented textures at an early stage already. These authors reported a new and rare type of orientation selective neurons in areas V1 (4\%) and V2 (1.6\%) of the visual cortex of monkeys which they named grating cells. Within area V1 these cells were mostly found in upper layers II, III and IVb. They responded strongly to bar gratings, but weakly or not at all to single bars or edges. They also preferred spatial frequencies of about 9 cycles/degree on average with a tuning width of one octave roughly. Grating cells were narrowly tuned to orientation. Most importantly, their response activity depended critically on the number of cycles of the gratings. They showed a strongly nonlinear response behaviour with a lower threshold of 4 grating cycles and reached saturation already with 8 grating cycles, on average. Furthermore most of the grating cells showed contrast switching with rather low contrast thresholds. They were not activated by harmonic components, as the spatial frequency response curves to sine wave, square wave and line gratings were all similar. Additionally they gave unmodulated responses to drifting gratings, were unselective for direction of motion and showed length summation and even strong end-stopping. In summary, the main purpose of these cells seems to be a fast and reliable detection of periodic patterns. This is an essential task in the perception of textures and, possibly, in theperception of depth (Sakai and Finkel, 1997). Hence, the preferences for single or multiple edges both seem important variables of feature extraction at early visual information processing stages. The lateral grouping of these response properties in the superficial layers of striate cortex is certainly an important issue to be investigated further. Grating cells are found in the same cortical area as simple cells. However, it is yet unknown which cells provide input to grating cells.

As Blasdel and Obermayer (Blasdel and Obermayer, 1994) pointed out recently, pyramidal cells - engaged in the full cortical circuitry including inhibitory interneurons - are responsible for the maps of orientation preference and they are 
also the cells most likely to use this information. If so the information flow processed by these cells must be highly constrained by the extent of their dendritic arbors amounting to $200-300 \mu \mathrm{m}$ in diameter. As information is only available locally, Blasdel and Obermayer hypothesized that cortical cells, in order to be good texture analysers, should group preferentially around pinwheel vortices of the underlying orientation map. While pyramidal cells within linear zones could only code about half of all orientations (due to their receptive field size of about $200-300 \mu \mathrm{m}$ ), neurons close to a \pm 1 / 2 vortex are influenced by all orientations and thus might be better suited for perception of textures. But as cortical neurons of monkeys in the center of pinwheel vortices are only poorly orientation selective and close neurons around the vortices encode the whole range of orientation preferences, it is unclear, whether they can provide enough output for the activity-dependent self-organization of grating-cells, which in contrast show strong and narrowly tuned orientation preferences generally.

In a recent study (Brunner et al. 1998) we pursued the hypothesis that grating cells form during development in an activity dependent fashion in the upper cortical layers receiving afferent input from cortical simple cells only. These simple cells show orientation preferences which change smoothly across the cortical surface. Hence within iso-orientation domains neighboring cells respond to closely similar oriented visual stimuli. Their output activities may thus drive the emergence of grating cells during postnatal development. Due to their high specificity to spatially periodic stimulus patterns it seems plausible that a sufficient number of simple cells has to provide strong input to any target cell to drive the latter to become a grating cell proper. This condition is easily met by simple cells from iso-orientation domains all having about the same orientation specificity under stimulus conditions provided by periodic textures. The latter certainly form an important but infrequently occuring class of biological stimuli.

In this study we will extend our recently developed incremental Hebbian learning model (Burger and Lang, 1999; Bauer et al., 1998) with plastic afferent as well as intracortical synaptic couplings to simulate the activity-dependent selforganization of periodic-pattern-selective cells driven by spatially periodic visual stimuli with varying orientation, phase and spatial frequency. Our main purpose is to study the necessary conditions for the emergence and maturation of only few model grating cells as well as their spatial organization relative to the underlying orientation map formed by cortical simple cells. Furthermore we are interested in the influence of additional lateral input onto the highly characteristic response properties of any single grating cell and, most importantly, on the way the range and strength of the lateral coupling structure will control the number and spatial layout of emerging grating cells. As most experimentally detected grating cells received input from one eye only a monocular model architecture may suffice.

\section{A Simulation Model of Grating Cells}

According to the two-phase learning paradigm proposed recently (Brunner et al., 1998) we first have to consider the development of feature maps driven by random input stimuli. Following the seminal papers by Linsker (1986) and Miller et al. (1989) a rather simple incremental Hebbian learning model for the prenatal development of receptive field structures, orientation preference and selectivity of simple cortical cells has been proposed recently (Burger and Lang, 1999; Burger and Lang, 1997). It considers the activity dependent adaptation of afferent as well as lateral synaptic coupling strengths. It is used in this study to produce realistic receptive field structures and orientation maps. During a second learning phase the activity-dependent development of periodicpattern-selective cells in upper cortical layers is simulated.

According to our recently discussed supposition (Brunner et al., 1998) model grating cells receive their input from model cortical simple cells within iso-orientation domains of the model orientation map which code similar preferred stimulus orientations. In this study we have shown that if a sufficient number of these orientation specific simple cells show strong activity in response to a grating stimulus the resulting postsynaptic potential together with a threshold nonlinearity suffices to stimulate the emergence of periodic-pattern-selective cells in subsequent cortical layers. The current investigation will pursue the hypothesis that the 
spatial self-organization of grating cells will be controlled by adaptable lateral synaptic couplings. The modification of the characteristic response properties by plastic lateral couplings between emergent grating cells will be investigated, too. In summary, we will explore the twofold hypothesis, that adaptive afferent synaptic couplings mainly control the emergence of grating cells and adaptive lateral synaptic couplings control their number and spatial layout with respect to the underlying orientation map.

Virtually nothing is really known about anatomical and developmental details of the functional architecture concerning grating cells. Even the question of which cells provide input to grating cells is a largely unresolved issue. From their contrast sensitivity Von der Heydt et al. (1992) argued that some of the grating cells receive input from the parvocellular stream, which in monkeys projects from layer $I V c_{\beta}$ to the blob regions of layers II/III, whereas others receive input from the magnocellular stream. The latter projects from layer $\mathrm{IV} c_{\alpha}$ to layer IVb and then to the interblob regions of layers II/III (Kandel et al., 1996). Whereas cells in layer IVc of monkeys do not seem to show strong orientation specificity, those in layer $\mathrm{IVb}$ do.

In our investigation we will consider a rather more simplified model architecture consisting of four model layers comprising the early visual path-

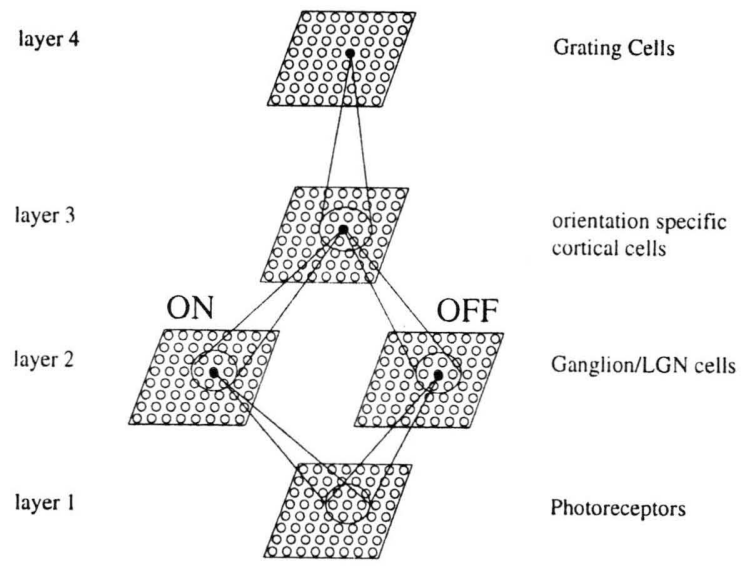

Fig. 1. Architecture of the neural network model. Visual stimuli are passed on from the photoreceptors via the ganglion/LGN layers to layer IV of the primary visual cortex. This layer serves as input layer for the emerging grating cells. way. The model layers may loosely be associated with the photoreceptor layer as the input layer, model layer 2 which comprises separate ON and OFF layers with ganglion cell and LGN cell layers, model layer 3 with cortical layer IV and model layer 4 as layers II/III of the visual cortex.

Within model layer 4 a target cell at $\vec{r}_{a}$ receives its afferent input from model layer 3 neurons at $\vec{r}_{s}$ within a localized square receptive field (RF) of length $r_{\Omega 0}$. The circular arbor function $\mathrm{A}_{G}\left(\vec{r}_{a}, \vec{r}_{s}\right)$ specifying the connection density of afferent axonal arbors and the grating cell dendrites is approximated simply by a step function with the same radius.

The output activities of model layer 3 neurons are transformed via a threshold nonlinearity with threshold $\Theta$ according to:

$s_{\Theta}\left(\vec{r}_{s}\right)=\left\{\begin{array}{cc}0 & \text { for }\left|s\left(\vec{r}_{s}\right)\right|<\Theta \\ \left|s\left(\vec{r}_{s}\right)\right| & \text { for }\left|s\left(\vec{r}_{s}\right)\right| \geq \Theta .\end{array}\right.$

The postsynaptic potential $h\left(\vec{r}_{a}, t\right)$ of a target cell at $\vec{r}_{a}$ is then given by a weighted sum of normalized afferent input activities $s_{n}\left(\vec{r}_{s}\right)=\frac{s_{\Theta}\left(r_{s}\right)}{\max _{s}\left(s_{\Theta}\left(\vec{r}_{s}\right)\right)}$ of the model layer 3 neurons within a square receptive field of length $\vec{r}_{\Omega 0}$ and the lateral input of other model layer 4 at $\vec{r}_{a^{\prime}}$ within the lateral coupling radius $r_{l 0}$ :

$$
\begin{aligned}
h\left(\vec{r}_{a}, t\right)= & \sum_{\substack{\vec{r}_{s} \\
\left|\vec{r}_{s}-\vec{r}_{a}\right| \leq r_{S O}}} A_{G}\left(\vec{r}_{a}, \vec{r}_{s}\right) \Omega\left(\vec{r}_{a}, \vec{r}_{s} t\right) s_{n}\left(\vec{r}_{s} t\right)+ \\
& +\sum_{\substack{\vec{r}_{a^{\prime}} \\
\left|\vec{r}_{a^{\prime}}-\vec{r}_{a}\right| \leq r_{10}}} l\left(\vec{r}_{a}, \vec{r}_{a^{\prime}, t}\right) h\left(\vec{r}_{a^{\prime}}, t\right),
\end{aligned}
$$

where $\Omega\left(\vec{r}_{a}, \vec{r}_{s}, \mathrm{t}\right)$ represents the afferent coupling strength between a model layer 3 neuron at $\vec{r}_{s}$ and a model layer 4 target cell at $\vec{r}_{a}$ and $l\left(\vec{r}_{a}, \vec{r}_{a^{\prime}}, t\right)$ represents the sum of the excitatory and inhibitory lateral coupling strengths between two model layer 4 cells at $\vec{r}_{a}$ and $\vec{r}_{a^{\prime}}$, respectively.

The activity $a\left(\vec{r}_{a}, t\right)$ of any target cell at time $t$ is then obtained via a nonlinear transformation of the postsynaptic potential $h\left(\vec{r}_{a}, t\right)$ according to a stepwise linear function $T\left(h\left(\vec{r}_{a}, t\right)\right)$ with an activation threshold $h_{1} \leq p_{1} \cdot N$ and a saturation threshold $h_{2}=p \cdot N$ with concomitant maximal cell activity $a_{\max }\left(\vec{r}_{a}, t\right)$ 
and number $N$ of afferents converging to the target cell.

$a\left(\vec{r}_{a}, t\right)=T\left(h\left(\overrightarrow{\mathrm{r}}_{a}, t\right)\right)= \begin{cases}\left(p_{2}-p_{1}\right) \cdot N & \text { for } h \geq h_{2}=p_{2} \cdot N \\ h\left(\overrightarrow{\mathrm{r}}_{a}, t\right)-p_{1} \cdot N & \text { for } h_{1}<h\left(\overrightarrow{\mathrm{r}}_{a}, t\right)<h_{2} \\ 0 & \text { for } h \leq h_{1}=p_{1} \cdot N .\end{cases}$

The afferent synaptic weights from model layer 3 cells to any target cell are now updated according to a Hebb-like learning rule according to

$\Delta \Omega\left(\vec{r}_{a}, \vec{r}_{s}, t \rightarrow t+1\right)=$

$\eta_{\text {hebb,aff }}^{G} a\left(\vec{r}_{a}, t\right) s_{n}\left(\vec{r}_{s} t\right)-\eta_{\text {dec }}^{G} \Omega\left(\vec{r}_{a}, \vec{r}_{s}, t \rightarrow t\right)$,

with $\eta_{\text {hebb,aff }}^{G}$ the learning step size and $\eta_{\text {dec }}^{G}$ the step size for a subtractive decay term which not only enables faster convergence but also suppresses the transformation to grating cells of all those model layer 4 cells which do not receive a strong enough input (i.e. $h \leq h_{1}$ ). Hence these cells remain cortical simple cells. The excitatory and inhibitory lateral couplings among the model layer 4 cells are updated by an anti-Hebbian and a Hebbian learning rule, respectively, using a constant sum constraint.

Hence the updated lateral excitatory coupling at time $t+1$ between model layer 4 cells at $\vec{r}_{a}$ and $\vec{r}_{a^{\prime}}$ is given by

$l_{e x}\left(\vec{r}_{a}, \vec{r}_{a^{\prime}}, t+1\right)=$

$$
\mathrm{C}_{e x} \cdot \frac{l_{e x}\left(\vec{r}_{a}, \vec{r}_{a^{\prime}}, t\right)-\eta_{\text {hebb,lat }} a\left(\vec{r}_{a}, t\right) a\left(\vec{r}_{a^{\prime},}, t\right)}{\sum_{\substack{\vec{r}_{a}^{\prime} \\\left|\vec{r}_{a^{\prime}}-\vec{r}_{a}\right| \leq r_{e x}}}\left[l_{e x}\left(\vec{r}_{a}, \vec{r}_{a^{\prime}}, t\right)-\eta_{\text {hebb,lat }} a\left(\vec{r}_{a,} t\right) a\left(r_{a^{\prime}}, t\right)\right]}
$$

whereas the inhibitory coupling can be written as

$$
\begin{aligned}
& l_{\text {in }}\left(\vec{r}_{a}, \vec{r}_{a^{\prime}}, t+1\right)= \\
& \mathrm{C}_{\text {in }} \cdot \frac{l_{\text {in }}\left(\vec{r}_{a}, \vec{r}_{a^{\prime}}, t\right)-\eta_{\text {hebb,lat }} a\left(\vec{r}_{a}, t\right) a\left(\vec{r}_{a^{\prime}}, t\right)}{\sum_{\substack{\vec{r}_{a^{\prime}} \\
\left|\vec{r}_{a^{\prime}}-\vec{r}_{a}\right| \leq r_{\text {in }}}}\left[l_{\text {in }}\left(\vec{r}_{a}, \vec{r}_{a^{\prime}}, t\right)-\eta_{\text {hebb,lat }} a\left(\vec{r}_{a}, t\right) a\left(r_{a^{\prime}}, t\right)\right]}
\end{aligned}
$$

using

$$
C_{e x}=\sum_{\substack{\vec{r}_{a^{\prime}} \\\left|\vec{r}_{a^{\prime}}-\vec{r}_{a}\right| \leq r_{e x}}} l_{e x}\left(\vec{r}_{a}, \vec{r}_{a^{\prime}}, t_{0}\right)
$$

and
$C_{\text {in }}=\sum_{\substack{\vec{r}_{a^{\prime}} \\\left|\vec{r}_{a^{\prime}}-\vec{r}_{a}\right| \leq r_{i n}}} l_{i n}\left(\vec{r}_{a}, \vec{r}_{a^{\prime}}, t_{0}\right)$

as the initial excitatory and inhibitory lateral coupling sums, respectively and $r_{e x}$ and $r_{i n}$ as the respective ranges of excitatory and inhibitory couplings.

During learning all synaptic weights have been updated synchronously. With these learning rules a periodic-pattern-selective cell may form in an activitydependent manner during development if the following conditions are met:

- it receives strong enough input from orientation specific simple cells $\left(s_{\Theta}\left(\vec{r}_{s}\right)>\Theta\right)$

- most of them are active at the same time responding strongly to optimal grating stimuli thus providing a large postsynaptic potential

- most of the neighboring model layer four cells are active and modulate the postsynaptic potential via lateral couplings.

The learning rule reinforces those synapses $\Omega\left(\vec{r}_{a}\right.$, $\vec{r}_{s}, \mathrm{t}$ ) whose corresponding pre- and postsynaptic activities reach a certain threshold controlled by the parameters $\Theta$ and $p_{1}$. If the presynaptic activity is below the activation threshold $\Theta$, the corresponding synaptic coupling strength will decay. Also if the postsynaptic potential at the grating cell does not exceed the activation threshold $h_{1}=p_{1} \cdot N$ of the activation function learning will not occur because the output activitiy is clamped to zero in this case.

For the afferent activity $s_{\Theta}\left(\vec{r}_{s}\right)$ to exceed the activation threshold $\Theta$ the driving stimulus has to be nearly optimal. In case of orientation specific cortical simple cells the latter would be provided by an optimally oriented bar stimulus, for example. Any suboptimally oriented stimulus even of high contrast would yield $s_{\Theta}\left(\vec{r}_{s}\right)<\Theta$, thus will not result in a strengthening of the corresponding weights $\Omega\left(\vec{r}_{a}, \vec{r}_{s}\right.$, t). Yet, for learning to occur, also the total summed input $\left(\vec{r}_{a}, \mathrm{t}\right)$ to the target cell proper has to exceed the threshold $h_{l}$. This will be possible only, if a sufficient number of afferent activities, driven by near optimal stimuli with sufficient contrast, exceed the threshold $\Theta$, too. This condition will neither be met with a single bar stimulus even of maximal contrast nor with any random input stimuli. If learning occurs the synaptic couplings will grow exponentially. This is why all coupling strengths converging to a given target cell need to be constrained to the intervall $\Omega\left(\vec{r}_{a}, \vec{r}_{s}, \mathrm{t}\right) \in[0,1]$. At the end of the second learning phase, all afferent couplings to the target 
cell will either have reached their maximal value $\Omega\left(\vec{r}_{a}, \vec{r}_{s}, t\right)=1$ or will be reduced to $\Omega\left(\vec{r}_{a}, \vec{r}_{s}, t\right)=0$ due to the decay term in Eqn. (4).The philosophy behind the anti-Hebbian vs Hebbian adaptation of the lateral couplings is the following. An anti-Hebbian adaptation of the excitatory couplings will, in the early stages of weight adaptation, provide additional input to any given target cell from neighboring output cells. This additional input will decay, however, in the course of development, hence will not force the emergence of large clusters of grating cells all encoding similar orientations. A Hebbian adaptation of the inhibitory couplings, instead, enhances the competition between the emerging grating cells and maximizes their mutual spatial distance. As an increasing inhibitory lateral input will reduce the total activity of any given grating cell, often the total postsynaptic potential will be suppressed below threshold. As a result the related target cell will not transform into a grating cell proper during the learning phase. This will ensure that, during the simulation, within an emerging cluster of potential grating cells only those with the strongest excitatory input should survive and encode the related preferred orientation of the grating stimuli. Hence different emerging grating cells will encode different orientations and they will be localized over different parts of the underlying orientation map.

\section{Simulated Response Behaviour of the Grating Cells}

The emergence and the development of a single grating cell has been investigated recently (Brunner et al., 1998) on the basis of suitable underlying orientation maps $(16 \times 16$ and $32 \times 32$ neurons). The activity-dependent self-organization of these orienation maps was simulated under developmental conditions with random, unoriented stimulus patterns driving cortical receptive field formation with segregated ON/OFF subfields and orientation map formation with iso-orientation domains, linear zones, fracture lines and pinwheel vortices. No further maturation of these maps while presenting oriented visual stimuli has been considered for simplicity.

The activity-dependent self-organization of the spatial layout of such periodic-pattern-selective cells was the main focus of the current investiga- tion. It has been simulated by adding a $4^{\text {th }}$ layer to the network and keeping the couplings between model layer 2 and model layer 3 constant. Using circularly symmetric receptive fields for the model layer 4 cells and initialing the afferent as well as the lateral couplings randomly, training was accomplished by presenting variable contrast bar gratings or patches of natural images as visual stimuli. The corresponding length dimension of the bars has always been chosen larger than twice the receptive field size in order to exceed the latter considerably. The orientation and phase have each been varied from $0^{\circ}$ to $180^{\circ}$ in steps of $10^{\circ}$. Spatial frequency has been varied by changing the width of bars and related interbar distances from 1 to 10 lattice units. The number of bars comprising the gratings ranged from 1 to 15 . All weights projecting onto the target cell have been updated synchronously after each stimulus presentation. Typically a total of about 10000 weight updates proofed necessary.

With a square receptive field of length $r_{w 0}=6$ and a circular arbor function with the same radius for systems of $32 \times 32$ cortical neurons, 113 afferent synaptic couplings from model layer 3 cells to any given target cell drive its development according to the learning rule given in Eqn. (4). Additionally, the lateral coupling radius was initialed with $r_{e x}=$ 2.0 and $r_{i n}=5.0$, respectively.

The lateral coupling sums for each grating cell were fixed to $C^{\text {ex }}=0.025$ and $C^{\text {in }}=0.36$, respectively. Because of the larger range of inhibitory couplings their total strength had to be larger in order to well balance the effect of the excitatory lateral interactions. The activation threshold was set to $\Theta=0.7$ and the lower and upper thresholds of the nonlinear transfer function were set to $p_{1}=$ 0.2 and $\mathrm{p}_{2}=0.5$, for convenience. During recall
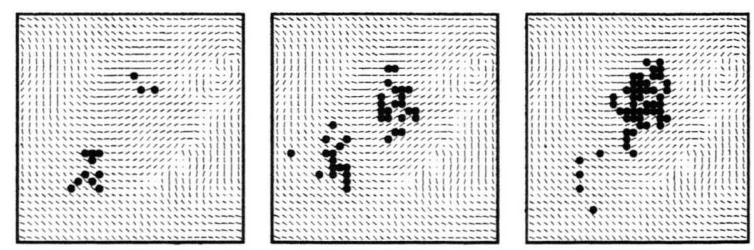

Fig. 2. Expanding the lateral excitatory radius from $r_{e x}=$ 1.0 (left), to $r_{e x}=3.0$ (middle) and $r_{e x}=5.0$ (right) the number of emerging grating-cells increases because of additional lateral input to thepostsynaptic potential of the grating cells. 
the above mentioned 1800 different grating stimuli were presented randomly and all output activities were phase averaged.

First the influence of the various parameters on the total number of mature grating cells and their spatial layout in relation to the underlying orientation map will be considered. Generally all synaptic coupling strengths are initialed with small random values. Figure 2 demonstrates the effect of an increasing radius of the lateral excitatory couplings with a constant but in any case larger inhibitory coupling radius. Starting from very few isolated grating cells expanding the range of excitatory lateral couplings leads to growing clusters of grating cells. Within any cluster all grating cells encode roughly the same orientation of the periodic pattern stimulus. Also it seems that no new centers of grating cell clusters are born while increasing the excitatory coupling radius. Rather the clusters build around the few isolated grating cells present at the start. It is to be noted that due to the learning process excitatory lateral couplings will eventually die out with only the inhibitory lateral couplings being left after convergence of the weight dynamics. Still their presence in the beginning suffices to create new grating cells which later on cannot be erased by the growing inhibitory couplings and their diminishing influence on the overall grating cell activity.

Increasing, instead, the radius of the inhibitory lateral coupling with a constant but in any case smaller excitatory coupling radius reduces the number of mature grating cells quickly and leads to a considerable dilution over the orientation map (see Fig. 3).
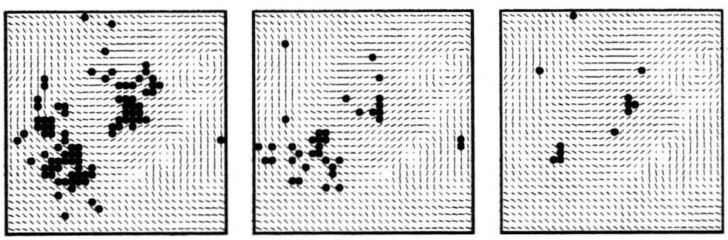

Fig. 3. An expansion of the lateral inhibitory coupling radius from $r_{i n}=4.0$ (left) to $r_{i n}=5.0$ (middle) and $r_{\text {in }}=$ 6.0 (right) leadsto a strongly decreasing number of grating cells.

Next the coupling radii of both the excitatory and the inhibitory couplings are fixed at a constant but equal range. Variation of the total inhibitory coupling strength while keeping the total excit- atory coupling strength constant but small results in a strong dilution of the grating cell clusters and a concomittant reduction in the number of mature grating cells as can be seen from Fig. 4 .
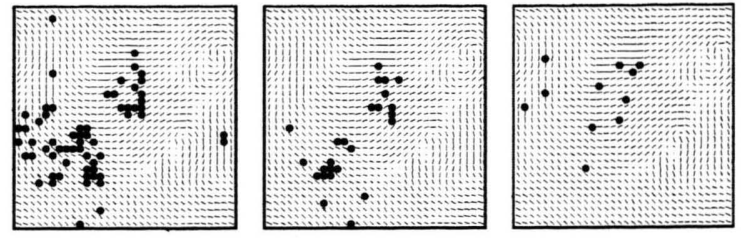

Fig. 4. Increasing the lateral inhibitory influce via the coupling strenght an according effect to an expansion of the inhibitory lateral coupling radius as shown in fig 3 can be observed.left: $C_{i n}=0.42$; middle: $C_{i n}=0.54$; right: $C_{\text {in }}=0.66$.

The effect is obviously similar to an increase in the range of inhibitory lateral couplings with their total strength held constant. Corresponding results are observed in the reverse situation, of course, as Fig. 5 demonstrates. Concerning suitable simulation parameters, biologically relevant developmental simulations of the self-organization of grating cells certainly have to respect a subtle balance between maximal robustness and minimal redundancy of information encoding (see Fig. 2-5).
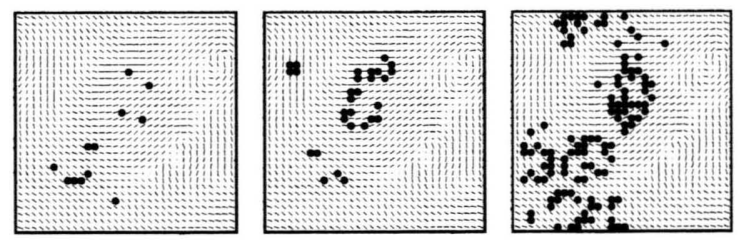

Fig. 5. A corresponding effect to an expansion of the lateral excitatory radius of the grating cells can be seen when increasing the total excitatory coupling strength from $C_{e x}=0.12$ (left) to $C_{e x}=0.24$ (middle) to $C_{e x}=$ 0.36 (right).

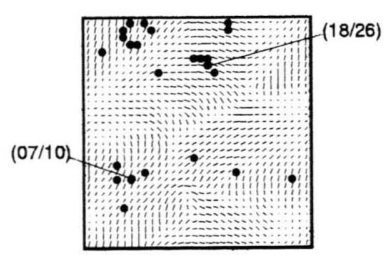

Fig. 6. Orientation map with grating cells. The excitatory lateral coupling radius was fixed to $r_{e x}=2.0$, the inhibitory to $r_{i n}=5.0$, respectively. The response properties of the neurons marked (07/10) and (18/26) will be examined in detail. 
Hence results displayed with the central graphs in figure 2-5 may most closely correspond to biologically relevant simulations.

Finally various characteristic response properties of the mature grating cells are investigated as concerns the modulation of their activity by additional lateral input.

In Fig. 6a typical oriention map with grating cells is presented. The defining response proper- ties of periodic-pattern-specific neurons (07/10) and $(18 / 26)$ are exemplified now:

Orientation and spatial frequency tuning curves (see Brunner et al., 1998 for a description of their determination) are slightly altered (see Fig. 7) due to the reduced response amplitude resulting from additional inhibitory lateral couplings to any given grating cell. The effect is to reduce somewhat the HWHM of these curves, leaving the preferred ori-
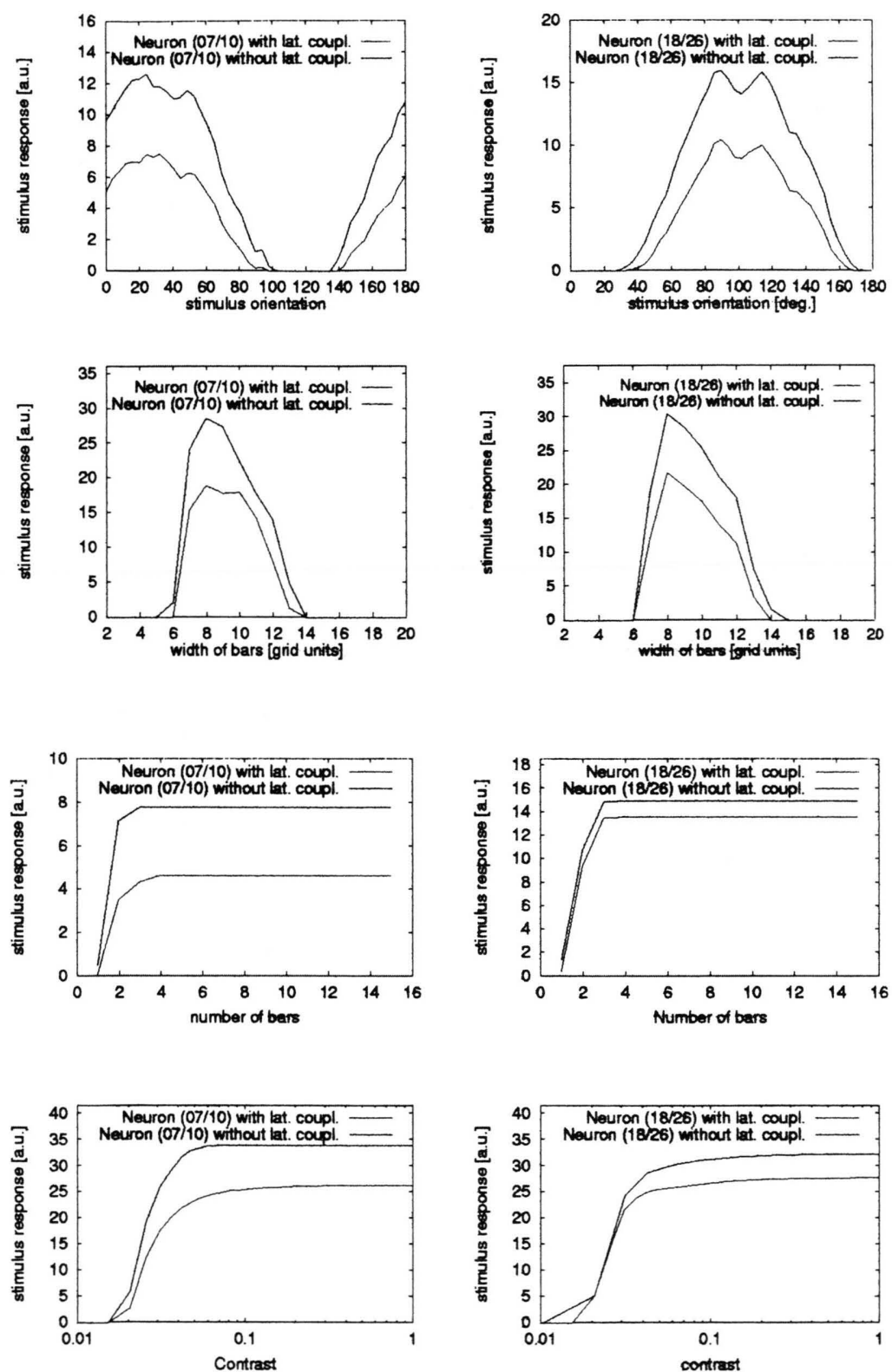

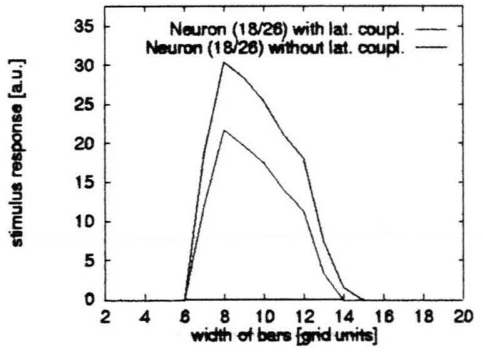

Fig. 7. Orientation and spatial frequency tuning curves of the model grating cells $(07 / 10)$ and $(18 / 26)$.
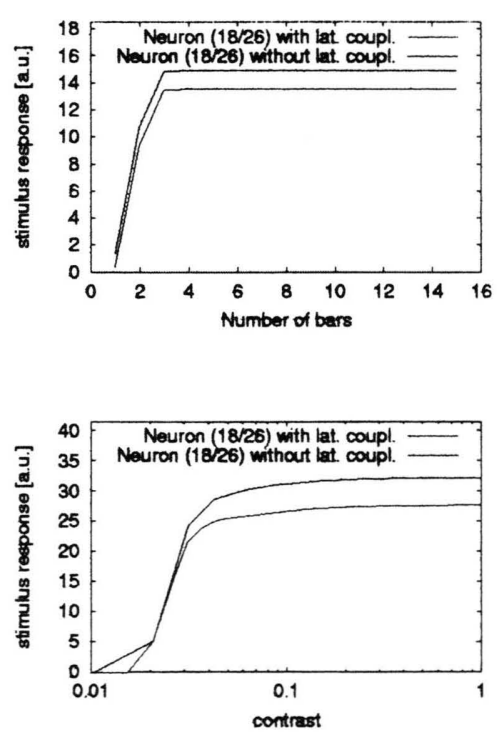

Fig. 8. Response functions to increasing number of bars and contrast of the model grating cells $(07 / 10)$ and $(18 / 26)$. 
entation and preferred spatial frequency unaltered, however.

Whereas the half width of spatial frequency tuning curves, shown in Fig. 7, corresponds well to experimentally determined values (1-1.5 octaves), the corresponding quantities seem fairly large in case of the orientation tuning curves. This issue has been discussed at length in Brunner et al. (1998). It is to be noted, however, that the simulated grating cells generally exhibit a considerably narrower orientation tuning than the corresponding cortical simple cells providing input to the grating cell under consideration. While the tuning width of model cortical simple cells amounts to $60^{\circ}$, model grating cells only show approximately $35^{\circ}$, which corresponds to a reduction of $40 \%$ in agreement with experimental findings (cortex: $20^{\circ}$, grating cell: $12^{\circ}$ ).

Concerning the response selectivity - quantified by the bar-grating index - to an increasing number of bars comprising the square wave grating at the preferred orientation and spatial frequency only slight alterations can be seen in Fig. 8 due to a somewhat reduced response amplitude. It demonstrates a strongly nonlinear response with an onset for gratings with $n^{0}=1$ and a saturation reached already with $n_{1}=4$ bars, yielding an index $\mathrm{n}=\frac{n_{0}}{n_{1}}=\frac{1}{4}=0.25$, in accordance with experimental values found by Von der Heydt et al. (1992). The additional inhibitory input slightly shifts the corresponding bar-grating index to higher absolute values, but the latter are still within the experimentally observed interval $0.08 \leq \mathrm{n} \leq 0.50$. Hence, the essential characteristic feature is almost unaltered and the typical stepwise linear response of real grating cells is well reproduced by our model grating cells.

As a new response characteristic the contrast switching, i.e. the almost constant response amplitude to a varying contrast of the gratings down to very low contrast values has been simulated also. Contrast $C$ has been defined by Von der Heydt as $C=\frac{L_{\max }-L_{\min }}{L_{\max }-L_{\min }}$ where $L_{\max }$ and $L_{\min }$ signify the maximum and minimum luminosity. Fig. 8 demonstrates a rapid increase of the grating cell activity after exceeding a very low threshold (approx. $1 \%-2 \%)$. Saturation is reached at about $3 \%-4 \%$, whereby the lower and upper limits of the nonlinear activation function (Eqn. (3)) determine the starting and saturation points of the contrast response behavior. This steep rise is only slightly smeared out due to the additional inhibitory input, which also lowers the saturated response amplitude. Still this defining response characteristic of grating cells remains almost unaltered in its general appearance. Again these results, and particularly the early response at very low contrast, correspond to the experimental data, obtained by Von der Heydt (1992).

\section{Discussion}

We have shown recently that a simple neural network model with a biologically motivated architecture can simulate some characteristics of the activity-dependent development of highly specific periodic-pattern-selective cells. The current investigation generalized this approach to a whole layer of grating cells and discussed the effect of plastic lateral synaptic couplings on the defining response properties of grating cells. Its main focus, however, concerned the activity-dependent spatial self-organization of emerging grating cells in relation to the underlying orientation map.

The model network consists of linear neurons up to model layer three, where the output neurons possess a semi-linear activation function with an activation threshold. It is in this layer, where orientation specificity maps form. In model layer four nonlinear output neurons with a sigmoidal activation function and normalized afferent activities and synaptic couplings were considered. Adaptive excitatory as well as inhibitory lateral synaptic couplings have been added and the resulting effects been investigated. Learning proceeds in two phases with different learning rules proper.

In the simulations orientation specific cells first form and self-organize into realistic orientation maps. This process is driven by random stimulus patterns and proceeds according to an incremental Hebbian learning rule with explicit stimulus presentation instead of using artificial input activity correlation functions. Loosely speaking this phase may correspond to the prenatal development of edge detectors and orientation maps in the visual cortex of mammals. Cortical simple cells are essentially linear analyzers of a part of a visual scene. 
As such, they basically extract information contained in the second order statistics of the input patterns. Since bandpass filtered white noise should show similar second order statistics than bandpass filtered natural scenes, the former is sufficient to drive their development.

During the second phase only the afferent excitatory synaptic couplings between orientation specific cells in model layer three and the output neurons are adapted according to a thresholded Hebb-like learning rule. All other afferent couplings in the layered network remain fixed. Output neurons received additional input from neighboring neurons through adaptable excitatory and inhibitory lateral synaptic couplings. During development the latter are updated according to a constant sum learning rule, which fixes the total strength of the excitatory as well as the inhibitory lateral couplings separately. Thereby inhibitory and excitatory couplings have been updated according to a Hebb- and an anti-Hebb-like rule, respectively. Hebbian adaptation of inhibitory couplings maximizes competition between emerging grating cells encoding similar texture characteristics. On the other hand anti-Hebbian learning of excitatory couplings favours the formation of small clusters of nearly identical grating cells providing a robust encoding of the whole range of texture characteristics contained in the stimulus pattern set.

With the learning rules proposed periodicpattern-selective cells do not form if only random stimuli are presented. Grating cells perform a highly nonlinear specialized task, signalling spatially periodic patterns characterized by higher order statistics. These higher order statistics can only be learned from structured visual stimulus patterns. Following this idea square wave stimuli with varying orientation $\left(0^{\circ}-170^{\circ}\right)$, phase $\left(0^{\circ}-180^{\circ}\right)$, spatial frequency (bar widths have been varied from 1-15 lattice units with a corresponding interbar distance) and number of bars (1-15) were used as training patterns.

Iso-orientation domains show a number of neighboring cortical neurons with similar orientation specificity. With the target cell receptive field centered there a grating pattern stimulus with proper orientation can evoke a sufficiently strong activity in all neurons within the receptive field. This strong afferent activity can drive any target cell in superficial cortical layers above threshold and evoke a strong response, too. With the proposed suitably thresholded Hebbian learning rule this target cell can develop into a periodic-patternselective cell in an activity-dependent manner.

The orientation and spatial frequency tuning, bar-grating specificity and contrast switching of the simulated model grating cells reproduce related aspects of the experimentally observed response behaviour very well. A single bar stimulus as well as higher Fourier components of grating stimuli will stimulate a small number of simple cells only leading to subcritical postsynaptic potentials. These stimuli do not contribute to learning, nor do they evoke any response in mature model grating cells due to high activation thresholds. The latter also account for the low spontaneous activity of grating cells.

Except for a generally somewhat better performance plastic lateral cortical couplings did not alter these simulated response characteristics much. However, they were essential as regards the activity-dependent spatial self-organization of emerging grating cells in relation to the underlying orientation map. Not surprisingly, grating cells formed whenever their receptive fields were centered over an iso-orientation domain of the related orientation map. This underlines that the emergence of model grating cells is driven mainly by afferent activities. Depending on the parameters of the lateral coupling network, more or less extended clusters of grating cells were distributed over the various iso-orientation domains thereby avoiding overlap of their receptive fields with any pinwheel vortices of the orientation map. Clearly there has to be a balance between robustness and redundancy of information encoding (Field, 1994; $\mathrm{Li}$ and Atick, 1994). Few isolated grating cells represent a rather fragile situation as the eventual loss of any grating cell means the related oriented texture will not be encoded furthermore. Large clusters of grating cells all encoding roughly similar oriented textures within any cluster represent a highly redundant information representation. In addition it seems plausibel that highly specialized feature detectors, which specialize on rather rare natural stimulus features, are expected to represent a rare type of cortical cells. The parameters chosen to study the various response characteristics of the grating cells in the presence of lateral 
input correspond to only few percent $(2 \%-4 \%)$ of mature grating cells in accord with experimental findings.

The observation that receptive fields of model grating cells are centered mainly within iso-orientation domains of orientation maps has to be seen in relation to the recent proposal of Blasdel and Obermayer (1994) concerning scene segmentation strategies in cortex. They hypothezise that the receptive field centers of texture discriminating cortical cells might group within $100 \mu \mathrm{m}$ distance around pinwheel vortices in order to locally en- code all texture orientations. With typical dendritic and local axonal arbors of $250 \mu \mathrm{m}$ each of these cells would indeed sample many different orientations. None of the simulated model grating cells had its receptive field centered at a pinwheel singularity. Rather the current model predicts that grating cells will avoid pinwheel singularities and group instead over iso-orientation domains. If one considers that periodic pattern selective cells may also serve a texture discriminating purpose then our model predictions are at odds with the Blasdel-Obermayer hypothesis.
Bauer C., Burger T., and Lang E. W. (1998), Ein neuronales Netzwerkmodell zur Selbstorganisation von Grating-Zellen in höheren Schichten des visuellen Kortex.Verhandl. DPG (VI) 33, 641.

Blasdel G. G. and Obermayer K. (1994), Putative strategies of scene segmentation in monkey visual cortex. Neural Networks 7, 865-881.

Brunner K., Kussinger M., Stetter M., and Lang E. W. (1998), A neural network model for the emergence of grating cells. Biol. Cybern 78, 389-397.

Burger T. and Lang E.W. (1997), A CBL network model with intracortical plasticity and natural image stimuli. Lecture notes in computersience 1327, 225-230.

Burger T. and Lang E. W. (1999), An incremental Hebbian learning model of the primary visual cortex with lateral plasticity and real input patterns. Z. Naturforsch. C54, 128-140.

Field D. J. (1994), What is the goal of sensory coding? Neural Computation 6, 559-601.
Kandel E. R., Schwartz J. H., and Jessell T. M. (eds) (1996), Neurowissenschaften. Spektrum Akademischer Verlag, Heidelberg.

Li Z. and Atick J. J. (1994), Toward a theory of the striate cortex. Neural computation 6, 127-146.

Linsker R. (1986), From basic network principles toneural architecture. Proc. Natl. Acad. Sci USA 83, 7508 $7512,8390-8394,8779-8783$.

Miller K. D., Keller J. B., and Stryker . M.P (1989), Ocular dominance column developement: Analysis and simulation. Science 245, 605-615.

Sakai K. and Finkel L. H. (1997), Spatial frequency analysis in the perception of persepctive depth. Network $\mathbf{8}, 355-352$.

Von der Heydt R., Peterhans E., and Dürsteler M. R. (1992), Periodic-pattern-selective cells in monkey visual cortex. J Neurosci 12(4), 1416-1434. 